\title{
Noncentrosymmetric Sulfide Oxide MZnSO (M=Ca or Sr) with Strongly Polar Structure as Novel Nonlinear Crystals
}

\author{
Ali H. Reshak 1,2,3,*, Nada M. Abbass ${ }^{4}$, Jiri Bila ${ }^{3}$, Mohd Rafie Johan ${ }^{2}$, Ivan \\ Kityk $^{5,6}$ \\ ${ }^{1}$ Physics department, College of Science, Basrah University, Basrah, Iraq \\ ${ }^{2}$ Nanotechnology and Catalysis Research Center (NANOCAT), University of Malaya, Kuala \\ Lumpur 50603, Malaysia \\ ${ }^{3}$ Department of Instrumentation and Control Engineering, Faculty of Mechanical Engineering, CTU in \\ Prague, Technicka 4, Prague 6166 07, Czech Republic \\ ${ }^{4}$ Department of Chemistry, College of Science, Baghdad University, Jaderiya, Baghdad, Iraq \\ ${ }^{5}$ Department of Experimental Physics and Information-measuring Technology, Lesya Ukrainka Eastern \\ European National University, Voli Avenue 13, Lutsk, 43025, Ukraine \\ ${ }^{6}$ Institute of Optoelecorncis and Measuring Systems, Czestochowa Univeristy of Technology, Armii \\ Krajowej 17, Czestotchowa, Poland
}

\section{Supporting Information}

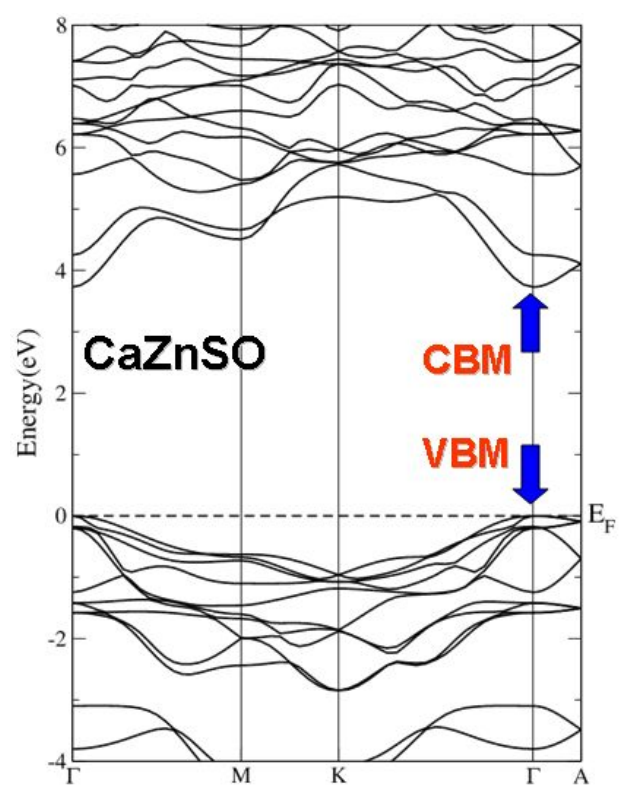

(a)

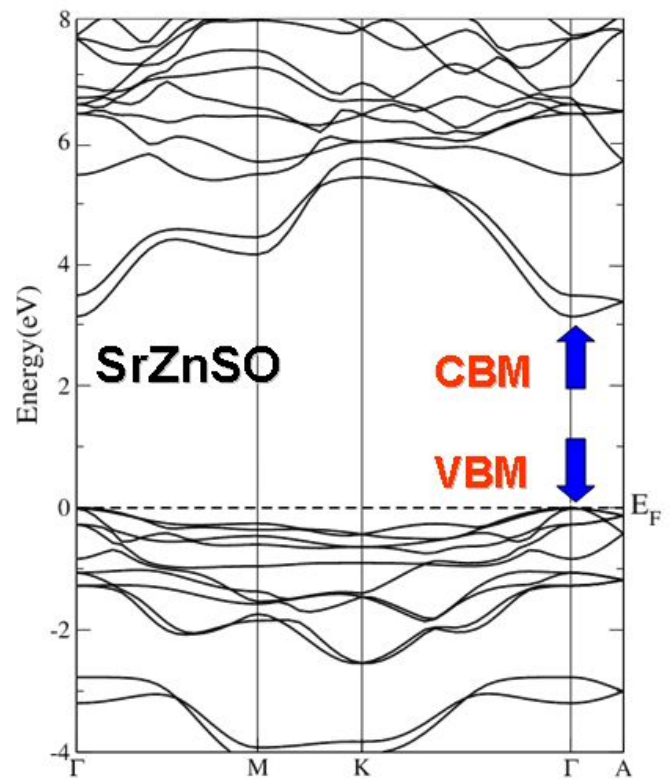

(b)

Fig. S1 


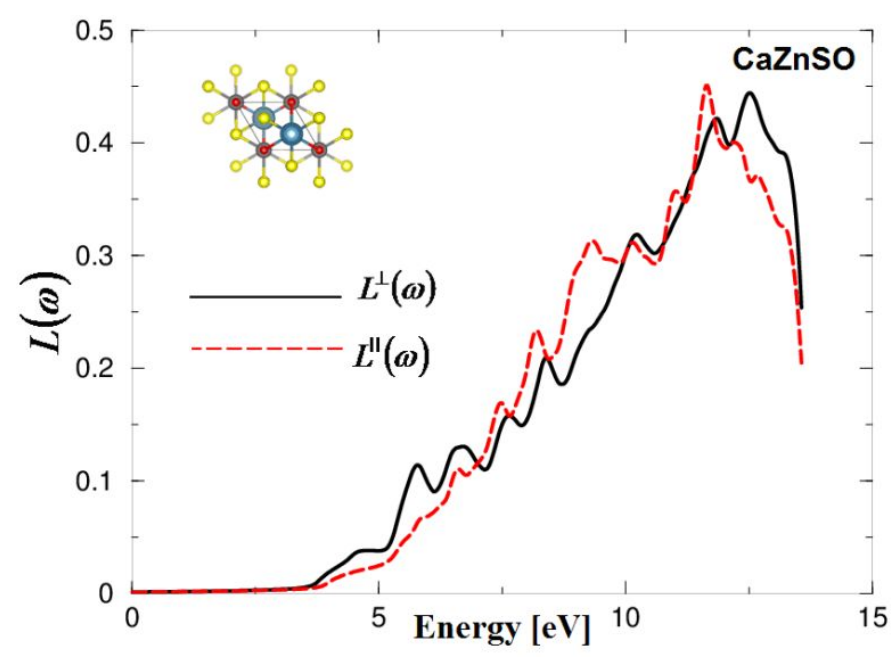

(a)

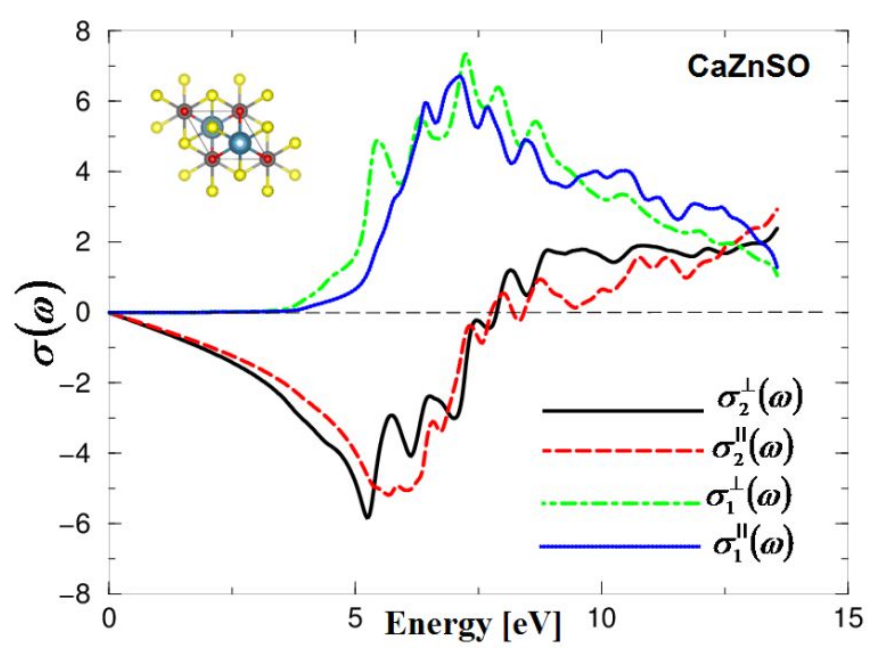

(c)

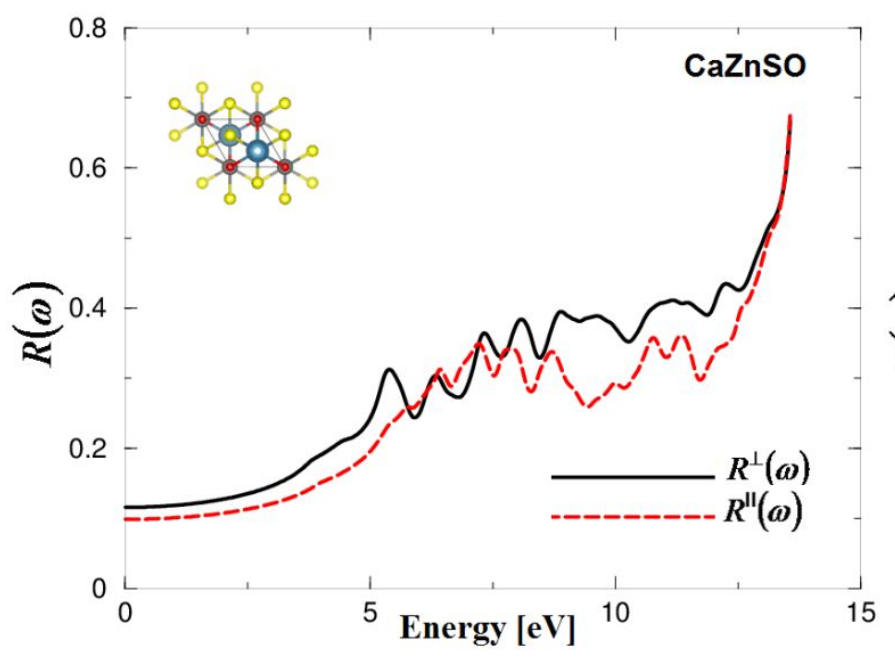

(e)

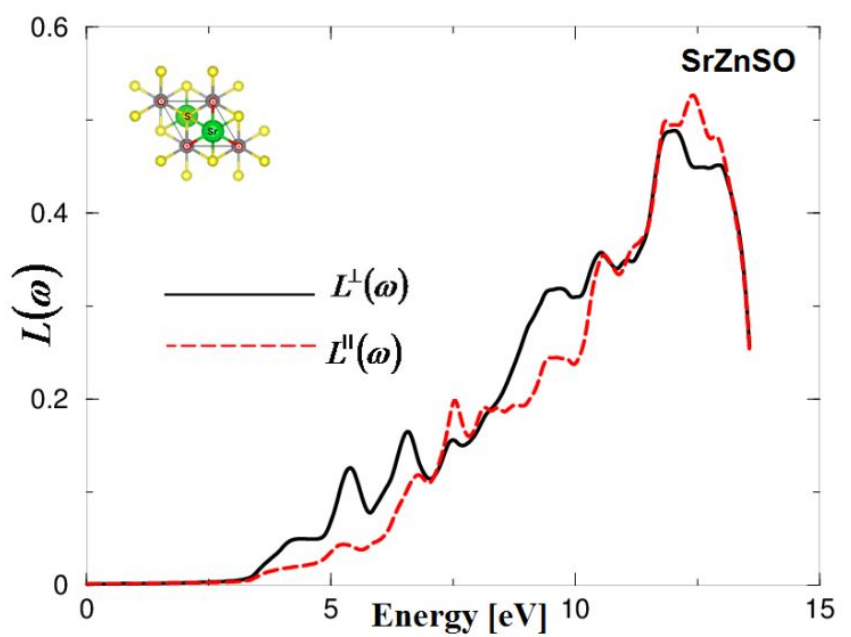

(b)

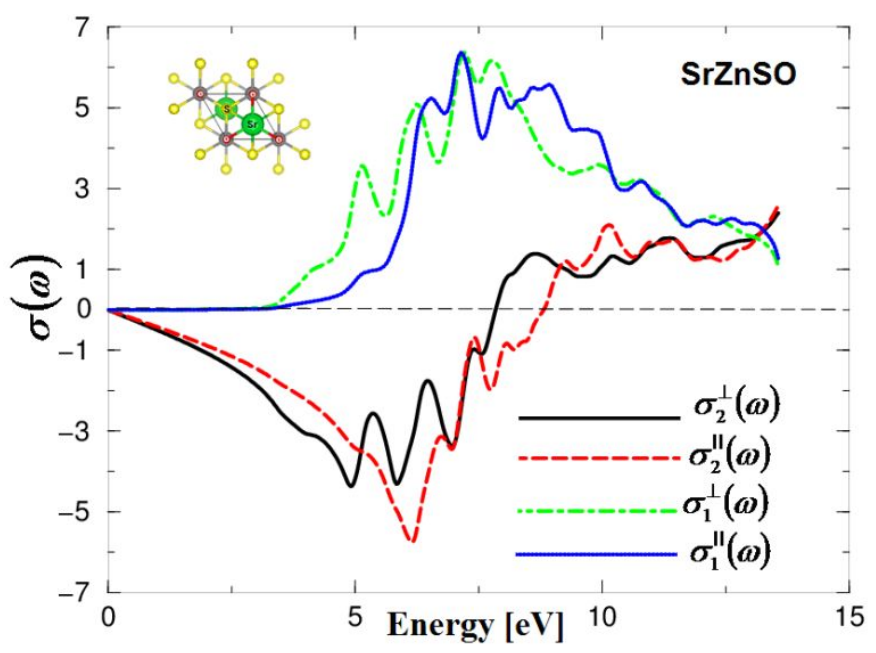

(d)

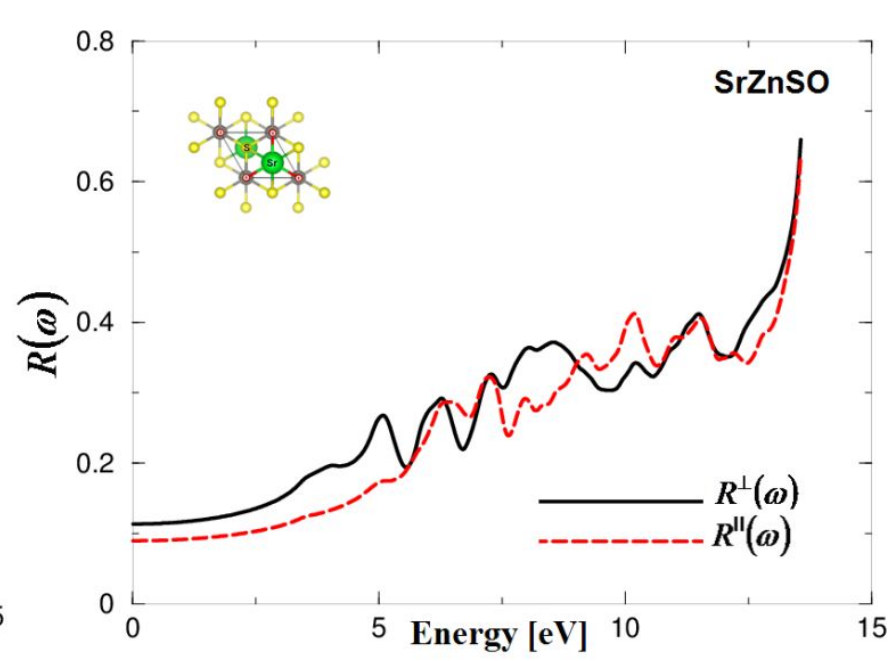

(f) 


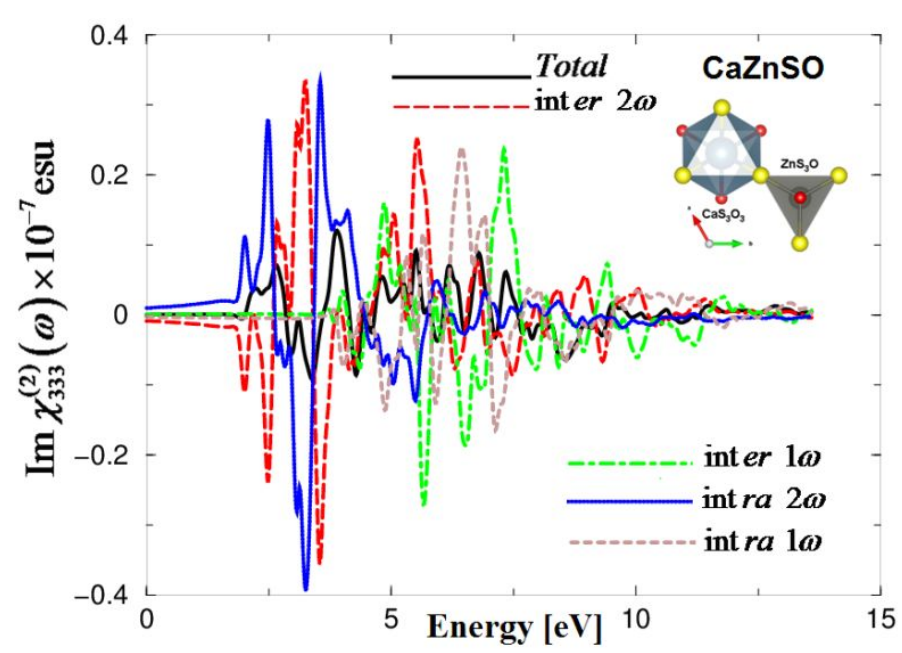

(g)

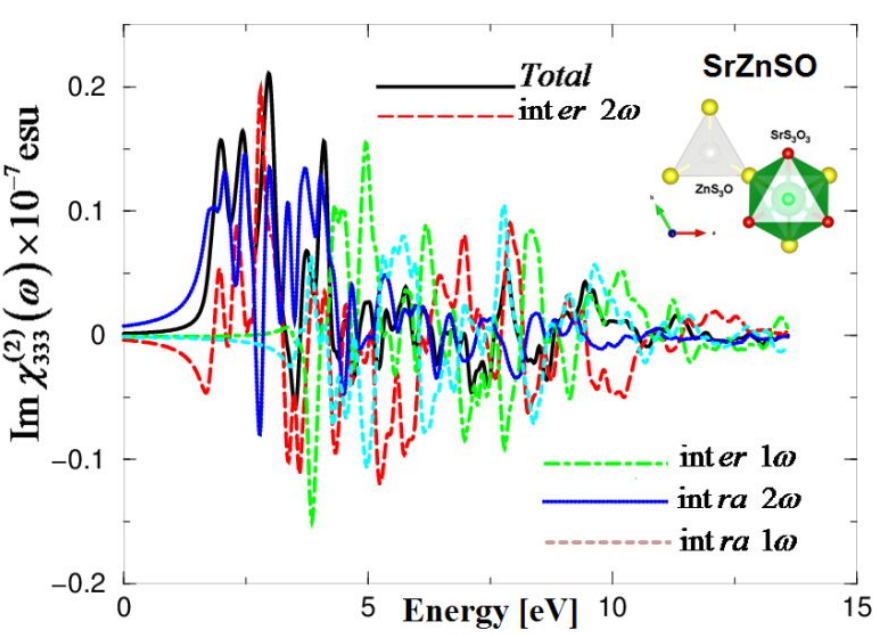

(h)

Fig. S2

\section{Figure captions:}

Fig. S1: Calculated electronic band structure of Sulfide Oxide $\mathrm{MZnSO}(\mathrm{M}=\mathrm{Ca}$ or $\mathrm{Sr})$.

Fig. S2: (a, b) Calculated loss function $L^{\perp}(\omega)$ (dark solid curve-back color online) and $L^{\prime \prime}(\omega)$ (light dashed curve-red color online); (c, d) Calculated $\sigma_{2}^{\perp}(\omega)$ (dark solid curveblack color online) and $\sigma_{2}^{\prime \prime}(\omega)$ (light dashed curve-red color online) along with Calculated $\sigma_{1}^{\perp}(\omega)$ (dark solid curve-blue color online) and $\sigma_{1}^{\prime \prime}(\omega)$ (light dashed curve-red online); (e, f) Calculated $R^{\perp}(\omega)$ (dark solid curve-black color online) and $R^{\prime \prime}(\omega)$ (light dashed curve-red color online); (e,f) Calculated total $\operatorname{Im} \chi_{333}^{(2)}(\omega)$ spectrum (dark solid curve-black color online) along with the intra $(2 \omega) /(1 \omega)$ (light solid curve-blue color online)/ (light dashed doted curve-cyan color online) and inter $(2 \omega) /(1 \omega)$ (light long dashed curve-red color online)/(light doted curve-green color online) -band contributions. 